\title{
Decision Trees for Human Activity Recognition Modelling in Smart House Environments
}

\author{
Veralia Gabriela Sánchez ${ }^{*}$, Nils-Olav Skeie \\ Department of Electrical Engineering, Information Technology and Cybernetics, \\ University of South-Eastern Norway (USN), Porsgrunn, Norway; *veralia.g.sanchez@usn.no
}

SNE 28(4), 2018, 177-184, DOI: 10.11128/sne.28.tn.10447 Received: November 27, 2018; Revised December 6, 2018; Accepted: December 10, 2018

SNE - Simulation Notes Europe, ARGESIM Publisher Vienna, ISSN Print 2305-9974, Online 2306-0271, www.sne-journal.org

Abstract. Human activity recognition in smart house environments is the task of automatic recognition of physical activities of a person to build a safe environment for older adults or any person in their daily life. The aim of this work is to develop a model that can recognize abnormal activities for assisting people living alone in a smart house environment. The idea is based on the assumption that people tend to follow a specific pattern of activities in their daily life. An open source database is used to train the decision trees classifier algorithm. Training and testing of the algorithm is performed using MATLAB. The results show an accuracy rate of $88.02 \%$ in the activity detection task.

\section{Introduction}

Human activity recognition modelling (HAM) in smart environments is an important area of research. Smart houses are being developed to improve and ease the life of the inhabitant. The idea of implementing HAM is to recognize the activities of a person in order to adapt the house to its user $[1,2]$.

A smart house is defined as any living environment that has been carefully designed to support its inhabitant in carrying out daily activities, as well as to promote independent lifestyles [3].

People tend to follow a pattern in their daily live $[4,5]$. Therefore, it is possible to recognize the activities of daily life (ADL) a user performs, such as eating, toileting, bathing, dressing, etc. This recognition task is also known as human activity recognition (HAR).

Once the ADL recognition task is done, HAM can use the output from it to learn the pattern of the user and model the user's activities. The modelling has the potential to detect any deviation from the usual pattern.

Detecting abnormal activities has several applications including assisting older adults. In Norway, $38.5 \%$ of households with people aged 65 and over are living alone [6]. Hence, a smart house can help the older adult to remain living in their own home for as long as possible [7].

In this work, HAR is implemented using an open source database. The output of the HAR is used for the HAM. HAM generally refers to the task of modelling the person activity pattern together with time. Therefore, accurate activity recognition is a crucial part for good HAM.

Decision trees are used to develop the HAM. Decision trees are a probabilistic algorithm that is able to predict the next step or value by learning from data. An open dataset is used for training the model.

\section{Related Work}

Decision tree is a supervised learning method. This method has been used for several tasks in the field of pattern recognition and machine learning as a predictive model. The main goal is to predict the next value given several input variable.

Previous studies on pervasive environment using decision trees have been successfully implemented [8].

In smart house environments, an $80 \%$ accuracy was achieved using decision trees on 20 everyday activities in a research by Bao and Intille ([9]). Another research based on decision tree with good result for ADL is the work by Fan et al. ([10]).

\section{Design and Methods}

Figure 1 shows the methodology flow in this work. 


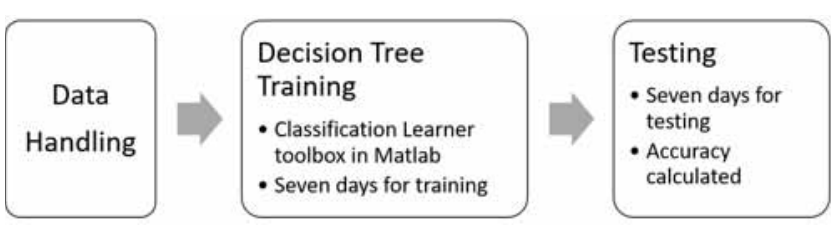

Figure 1: Methods.

\subsection{Decision trees}

Decision trees is a hierarchical model also known as classification and regression trees. They have the property of predicting response from data.

The attributes of the decision trees are mapped into nodes. The edges of the tree represent the possible output values. Each branch of the tree represents a classification rule, from the root to the leaf node [11].

\subsection{Software}

MATLAB is used in this study for developing the model. The classification learner toolbox was specifically used for training the tree. The code from the classification learner toolbox was exported and saved for later use with any other dataset. Testing was also coded in MATLAB.

Finally, the program Wolfram Mathematica is used for the results plots presented in this article.

\begin{tabular}{|c|c|}
\hline Name & Value \\
\hline Setting & Apartment \\
\hline Number of Rooms & 4 Rooms + Hall/Entrance \\
\hline Number of labelled days & 14 days \\
\hline Labels (ADLs included) & $\begin{array}{l}\text { Leaving, Toileting, Show- } \\
\text { ering, Sleeping, Breakfast, } \\
\text { Lunch, Dinner, Snack, } \\
\text { Spare Time/TV, Grooming }\end{array}$ \\
\hline Number of sensors & 12 sensors \\
\hline Sensors & $\begin{array}{l}\text { PIR: Shower, Basin, Cook- } \\
\text { top } \\
\text { Magnetic: Maindoor, } \\
\text { Fridge, Cabinet, Cupboard } \\
\text { Flush: Toilet } \\
\text { Pressure: Seat, Bed } \\
\text { Electric: Microwave, } \\
\text { Toaster }\end{array}$ \\
\hline
\end{tabular}

Table 1: ADLs Database.

\subsection{Dataset}

An open dataset is used in this study. The dataset has been previously used in other research and is known in the HAR field [12]. The dataset is named "Activities of Daily Living (ADLs) Recognition Using Binary Sensors Data Set" and is available for download at [13]. The purpose of using an open dataset is to obtain unbiased results.

The dataset consists of annotated ADLs collected by two different users living on a daily basis in a smart house. The activities in the dataset were manually labelled by the users. Table 1 presents the dataset attributes.

Two instances of data exist corresponding to each user living in the smart house. One dataset of 14 days (OrdonezA), and the second dataset of 21 days (OrdonezB). The first dataset data is depicted in Figure 2. The first dataset is used this work for creating and testing the model. The second dataset (OrdonezB) is implemented later in order to test the model with a different dataset.

Data Handling The variables used from the dataset are "Date", "Time", "Activity", and "Room". Another variable named "position" was added to improve the recognition task. This variable position correspond to one of the three following values: laying, sitting, standing.

Table 2 depicts the first day from the dataset. The dataset is in a text file format.

In order to model the decision tree, a sample was drawn from the dataset. All the 14 days in the dataset were stopped when the activity leaving was found. Seven days were used for training and seven days were used for testing.

The dataset text values were coded to numbers in order to develop the MATLAB code. Table 3 shows the rooms with their respective codes.

Numbers were also assigned to the activities to make the learning and decoding process more feasible.

Table 4 shows the activities with the assigned codes.

Table 5 shows the coding used for the position values.

A total of 9 activities, 5 rooms, and 3 positions are used. 

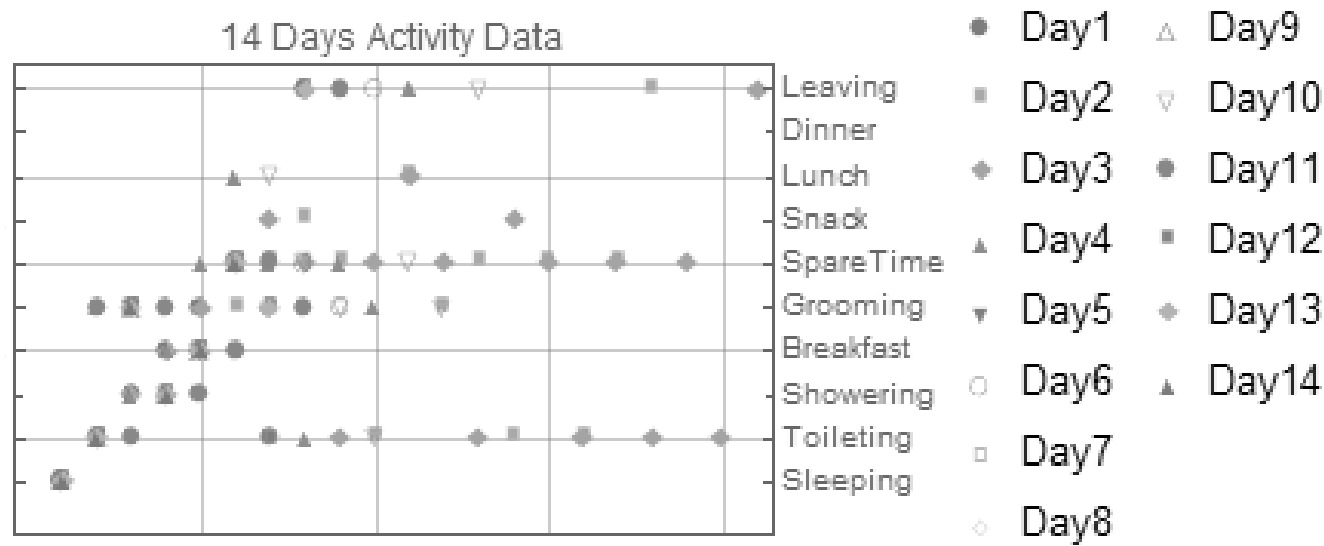

Figure 2: Activities Dataset Graph.

\subsection{HAR modelling (HAM)}

HAM refers to the modelling of the behaviour or activity of the person. Behaviour is regarded as an activity with duration, i.e. the time elapsed from start to end of an activity and time of day [14]. For example, a behaviour can be having breakfast, which consist of opening the refrigerator, cooking, sitting and eating breakfast. This set of activities are given in a time span (time elapsed from start to end), and usually in the morning (time of day).

Normal and abnormal activity and behaviour can be detected in a smart house by analysing both, the activity and the time. Abnormal activity detection main purpose is to warn a member of the family or caretaker whether something is wrong with the person. This can be regarded as anomaly. "Anomaly detection refers to the problem of finding patterns in data that do not conform to expected behavior" [15].

\section{Experiments}

The dataset used in this study is available in a text file only. Therefore, the dataset was exported to an excel file. The dataset contains 14 days of data in total. Two files were created, one for training and one for testing. Random numbers was generated in MATLAB with the randperm function to randomly select seven days for training. The numbers selected according to the random generator were days: 12, 10, 5, 14, 1, 7, and 6. Hence, these days were use for training. The remaining days $(2,3,4,8,9,11,13)$ were used for testing.

A new variable called duration was added. The vari- able duration was calculated using the time data from the dataset and consist of the time spent in each activity, from start to end of each activity. The duration value was calculated in seconds.

In the excel file, the text values of the dataset were coded to numbers. The variables room, position and activities were coded as explained in Section 2.3. The room values were coded to numbers from 1 to 5 . The position values coded to numbers from 1 to 3 . The $a c$ tivity values were coded to numbers from 1 to 9 .

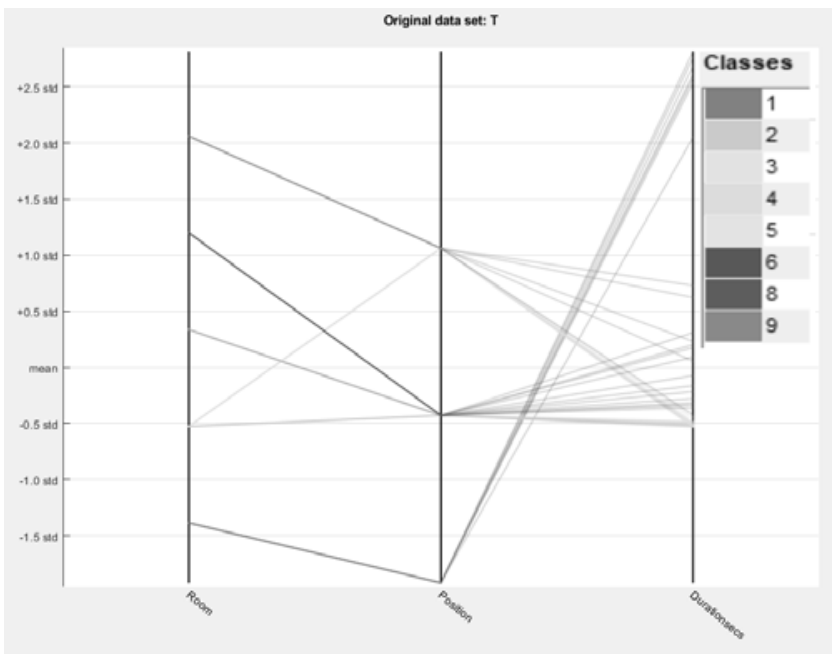

Figure 3: Parallel coordinated plots.

Both excel files were imported to MATLAB as table data type. The training was performed using the integrated classification learner toolbox. The variables used for training the decision trees are the room, position, and duration. The output variable is the activity 


\begin{tabular}{|c|c|c|c|c|}
\hline Date & StartTime & EndTime & $\overline{\text { Activity }}$ & Room \\
\hline 28-11-11 & $02: 27: 59$ & 10:18:11 & Sleeping & Bedroom \\
\hline $28-11-11$ & $10: 21: 24$ & $10: 23: 36$ & Toileting & Bathroon \\
\hline $28-11-11$ & $10: 25: 44$ & 10:33:00 & Showering & Bathroon \\
\hline $28-11-11$ & $10: 34: 23$ & 10:43:00 & Breakfast & Kitchen \\
\hline $28-11-11$ & 10:49:48 & $10: 51: 13$ & Grooming & Bathroon \\
\hline $28-11-11$ & 10:51:41 & 13:05:07 & $\begin{array}{l}\text { Spare } \\
\text { Time }\end{array}$ & $\begin{array}{l}\text { Living- } \\
\text { room }\end{array}$ \\
\hline $28-11-11$ & 13:06:04 & 13:06:31 & Toileting & Bathroon \\
\hline $28-11-11$ & 13:09:31 & 13:29:09 & Leaving & Hall \\
\hline $28-11-11$ & $13: 38: 40$ & $14: 21: 40$ & $\begin{array}{l}\text { Spare } \\
\text { Time }\end{array}$ & $\begin{array}{l}\text { Living- } \\
\text { room }\end{array}$ \\
\hline $28-11-11$ & $14: 22: 38$ & $14: 27: 07$ & Toileting & Bathroon \\
\hline $28-11-11$ & $14: 27: 11$ & 15:04:00 & Lunch & Kitchen \\
\hline $28-11-11$ & 15:04:59 & 15:06:29 & Grooming & Bathroon \\
\hline $28-11-11$ & 15:07:01 & $20: 20: 00$ & $\begin{array}{l}\text { Spare } \\
\text { Time }\end{array}$ & $\begin{array}{l}\text { Living- } \\
\text { room }\end{array}$ \\
\hline $28-11-11$ & 20:20:55 & $20: 20: 59$ & Snack & Kitchen \\
\hline $28-11-11$ & $20: 21: 15$ & 02:06:00 & $\begin{array}{l}\text { Spare } \\
\text { Time }\end{array}$ & $\begin{array}{l}\text { Living- } \\
\text { room }\end{array}$ \\
\hline
\end{tabular}

Table 2: Day 1 example of the dataset.

data.

Figure 3 shows the parallel coordinated plots of the data. The variables room, position, and duration are plotted to show the relationship between them. According to Figure 3, it is possible to see that activities 3 and 5 (showering and grooming) follow almost the same path line in the graph. Also, activities 4 and 8 (breakfast and lunch) almost follow the same path line, with the duration barely different for each of the two activities. Activity 7, snack, was not found in the training dataset.

Once the tree is trained, testing is performed with the remaining seven days of the dataset: days $2,3,4$, $8,9,11,13$. The testing consists on using the variables room, position, and duration as input data. The response or output is the activity value. Each day from the testing dataset was tested and compared to the real data.

A new fictional test set was created in order to test the model with abnormal data, as showed in Table 6 . The test set consists of a fictional single day. The table shows that the duration of some of the activities were exaggerated. In addition, the position: lying of the first

\begin{tabular}{l|l}
\hline Name of Room & Number Assigned \\
\hline Bedroom & 1 \\
\hline Bathroom & 2 \\
\hline Kitchen & 3 \\
\hline Livingroom & 4 \\
\hline Hall & 5 \\
\hline
\end{tabular}

Table 3: House rooms and their code.

\begin{tabular}{l|l}
\hline Name of Activity & Number Assigned \\
\hline Sleeping & 1 \\
\hline Toileting & 2 \\
\hline Showering & 3 \\
\hline Breakfast & 4 \\
\hline Grooming & 5 \\
\hline Spare time/TV & 6 \\
\hline Snack & 7 \\
\hline Lunch & 8 \\
\hline Leaving & 9 \\
\hline
\end{tabular}

Table 4: Activities numbers and their code.

activity in the hall room should qualify as abnormal behaviour.

Result plots were obtained using the Mathematica software. The actual data and the predicted data for each of the testing days was copied to Mathematica and plots were coded to visually present the results.

Finally, the total computational time was measured.

\section{Results}

Figure 4 shows the trained decision tree. The decision tree model was able to classify seven out of nine activities in the dataset.

Figure 5 shows the number of observations for each of the activities. The true class is in the y-axis and the predicted class is in the $\mathrm{x}$-axis. It is possible to see that there are no observations in the training dataset for activity number seven (snack), and only one observation for activity number eight (lunch).

Most error counts in Figure 5 occurred in activities that are performed in the same room, such as showering and grooming (3 and 5). However, this number of 


\begin{tabular}{l|l}
\hline Name of Activity & Number Assigned \\
\hline Lying & 1 \\
\hline Sitting & 2 \\
\hline Standing & 3 \\
\hline
\end{tabular}

Table 5: Position numbers and their code.

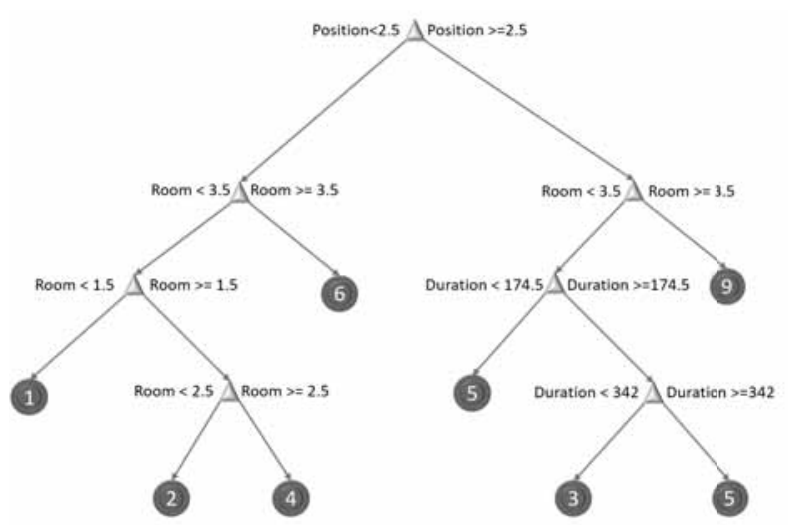

Figure 4: Trained Decision Tree. Red circles represent the activities.

observations with errors is low.

Figure 6 shows in percentage of success and errors prediction in the training data, called "positive predictive values false discovery rate". The highest false discovery is 50\% in the activity grooming (5). The model classified the activities showering (3) half of the times instead of the true class grooming (5).

For the activity grooming (5), the model had a false discovery rate of $22 \%$, classifying the activity showering (3) instead of grooming (5).

For the activity lunch (8), the model classified the activity breakfast (4) with a false discovery of $13 \%$.

Figure 7 shows the results of the test. Days 2, 3, 4,

\begin{tabular}{l|l|l|l}
\hline Room & Position & Durationsecs & Activity \\
\hline Hall & Lying & 10000 & - \\
\hline Bathroom & Sitting & 15000 & toileting \\
\hline Bathroom & Standing & 450 & grooming \\
\hline Living-room & Sitting & 9000 & spare time \\
\hline Living-room & Sitting & 9500 & spare time \\
\hline Hall & Standing & 412 & leaving \\
\hline
\end{tabular}

Table 6: Added fictional test day.

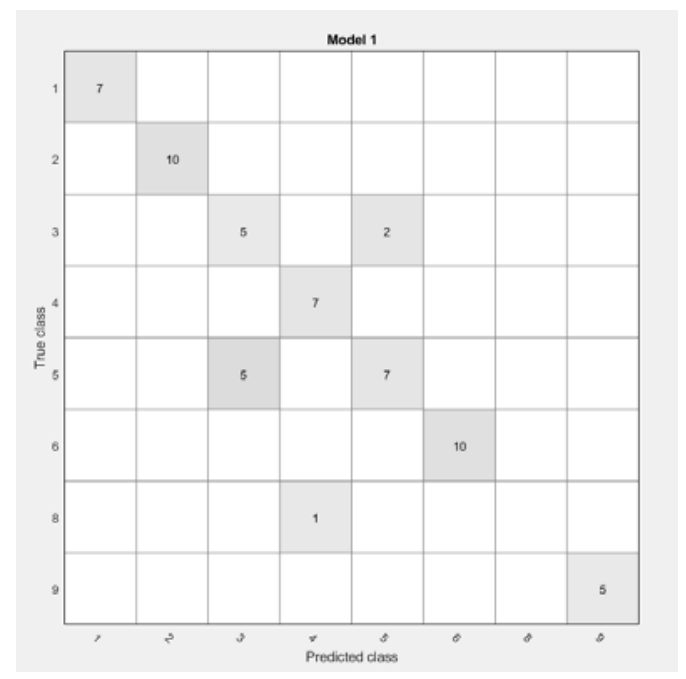

Figure 5: Number of observations.

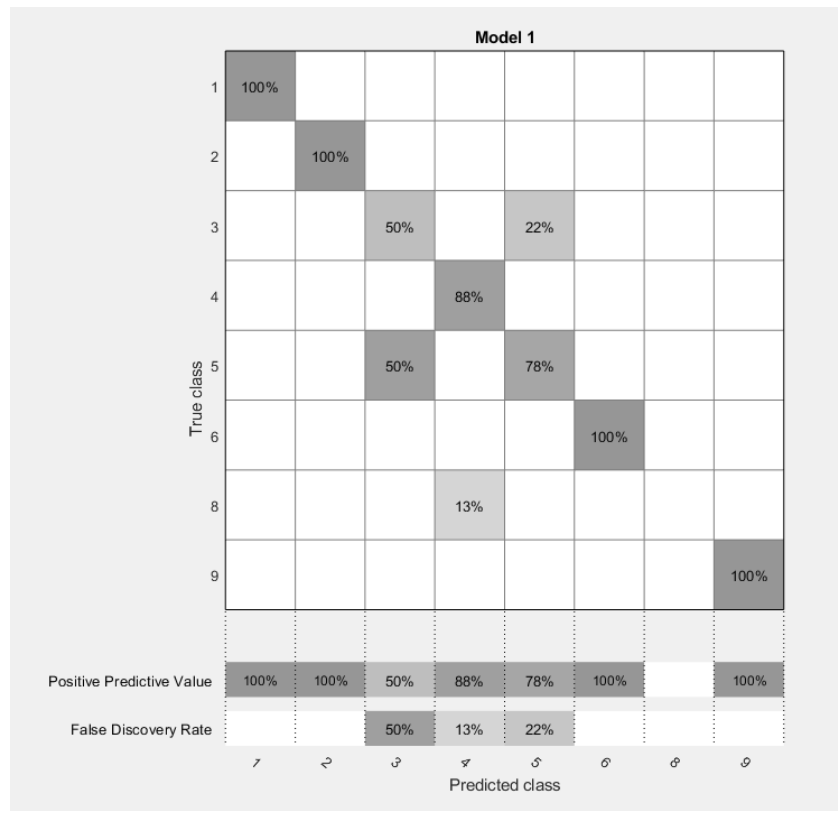

Figure 6: Positive predictive values and false discovery rates.

$8,9,11$, and 13 were used for testing. Some prediction errors were found when comparing the actual data with the estimated data.

Most of the errors were found between the grooming and showering activity, and between the breakfast and snack activities. A possible explanation for these prediction errors is that both of these activities are performed in the same room, bathroom and kitchen, respectively.

Figure $7 \mathrm{~h}$ shows the results of the added fictional 
day with abnormal behaviour data. The model predicted the activity Spare Time instead of finding an abnormal behaviour in the first activity.

The true positive-false positive rate for each fo the predicted activities are shown in Table 7.

Finally, the total accuracy of the activity recognition task is $88.02 \%$. The computational time of the model, consisting of training and testing is around 3 seconds.

\begin{tabular}{l|c|c}
\hline Activity & True Positive & False Positive \\
\hline Sleeping & $100 \%$ & 0 \\
\hline Toileting & $100 \%$ & 0 \\
\hline Showering & $77 \%$ & $23 \%$ \\
\hline Breakfast & $98 \%$ & $2 \%$ \\
\hline Grooming & $94 \%$ & $6 \%$ \\
\hline Spare time & $100 \%$ & 0 \\
\hline Lunch & $36 \%$ & $64 \%$ \\
\hline Leaving & $100 \%$ & 0 \\
\hline
\end{tabular}

Table 7: True positive, false positive rate for each activity.

\subsection{Test on second dataset}

The model was tested on the second dataset (OrdonezB) consisting of 21 days, also open source as described in Section 2.3. The purpose of this second test is to verify that the model works with any dataset.

In this test, the entire dataset was used, without sampling. The dataset was also processed as described in Section 3 . The values were coded to numbers. A total of 10 activities, 5 rooms and 3 positions were used.

The results showed that the model worked as well as in the experimental work (dataset Ordonez A). Like in the experimental work, minor mistakes were found in the prediction task corresponding to activities made in the same room. Namely bathroom and kitchen. Therefore, the model presented here is able to work with any dataset.

\section{Discussion}

In this work, decision trees are researched to perform human activity recognition modelling.

The decision tree classified seven out of nine activities. This is because there are no observation of activity snack, and only one observation for activity lunch in the training dataset. Therefore, the model could only classify seven activities in total.

Some predictions presented minor error rates. One possible reason for the these error rates is that there are rooms that allow different types of activities. Hence, the recognition task is more difficult. For example, in the room bathroom, three different activities are performed: showering, toileting and grooming. Thus, the model tends to predict the highest probability of the activity given the room bathroom.

This is the same case for the room kitchen, where activities breakfast, lunch and snack are performed. The activity lunch has an error rate of $64 \%$. From Figure 5 it is possible to see that there is only one observation of the activity lunch. Therefore, the model would hardly predict this activity. Instead, the model predicts the activity breakfast, because it has the highest probability.

Decision trees are probabilistic algorithms and thus produces some errors in the prediction task. As any probabilistic algorithm, decision trees will always chose the highest probability according to the trained data.

In general, the finding suggest that decision trees are a good tool for HAR with $88.02 \%$ accuracy. However, for the HAM, the model does not detects abnormal behaviour as well as it does HAR. When a fictional single day test set was created to check the performance on an abnormal day, the model did not meet the expectations in the anomaly detection task.

The most probable reason for this is that decision trees do not always enforce to check every variable before estimating a results. Consequently, in the added fictional test day with room hall and position lying, the tree predicted the activity spare time. Thus, the tree does not check for yhe other variables of room, nor $d u$ ration. The model should have detected an abnormal behaviour in this scenario, since lying in the hall is not a normal activity, but a possible fall.

Another reason for the model not detecting abnormal behaviour is that abnormal situations need to be trained in decision trees. This means that all possible abnormal scenarios need to be learned a priori. As a result, the findings suggest that decision trees are not the best option for detecting abnormal activities or behaviour.

The model was also tested with the second dataset available (OrdonezB) to verify that the model is able to work with any dataset. The results obtained were similar to the experimental work. Minor mistakes were 


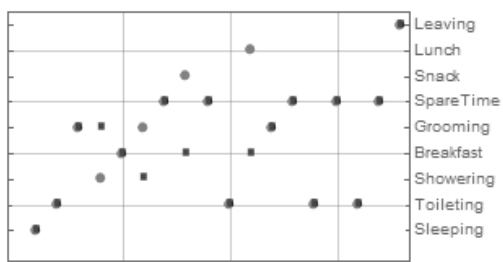

(a) Day 2 Results.

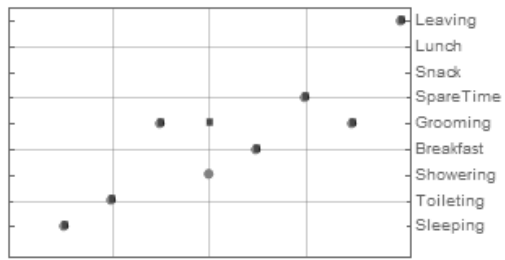

(d) Day 8 Results.

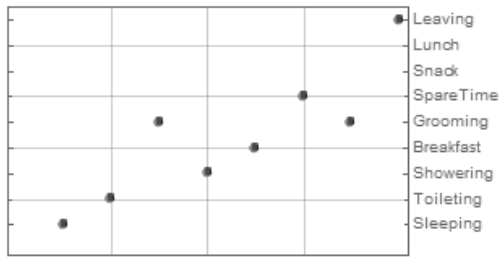

(g) Day 13 Results.

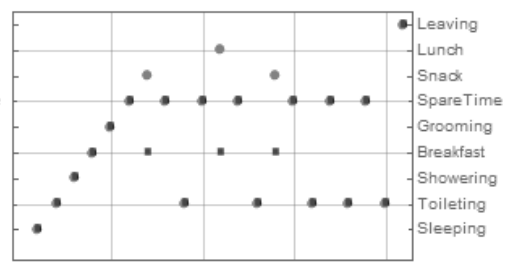

(b) Day 3 Results.

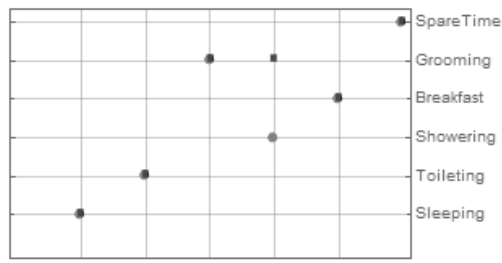

(e) Day 9 Results.

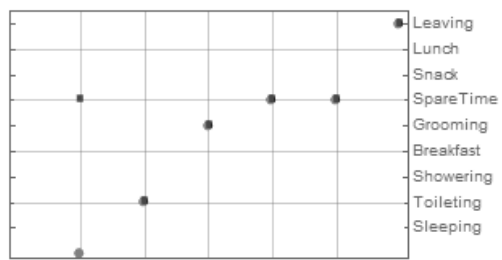

(h) Added fictional day results.

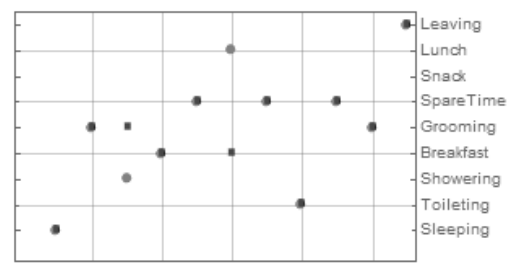

(c) Day 4 Results.

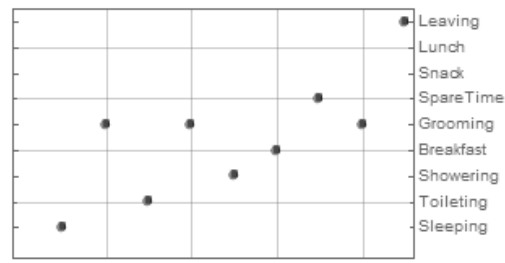

(f) Day 11 Results.

\section{- Actual Data \\ - Estimated Data}

Figure 7: Results.

found in activities performed in the same room.

Possible solutions for improving the model are more research on how to enforce the decision trees to check every single parameter. Another option could be to combine decision trees with another probabilistic method to increase the accuracy of the model.

Finally, HAM would ideally keep the activity history of the user in order to model the behaviour of the person. For example, if the user has followed the pattern wake up, toileting, grooming, showering and breakfast, the most normal behaviour would be not to repeat any of those activities again within a given frame time.

\section{Ethics in Smart Houses}

Smart house technology, like any other type of technology, can carry many ethical challenges. Therefore, a separate study has been carried at USN to address this topic. We consider that the ethical aspects are an important part of our research in smart house technology.

Among the main challenges found that smart houses present are cost-effectiveness, privacy, autonomy, in- formed consent, dignity, safety, and trust [16].

These challenges are central to keep in mind when developing a smart house system. Developers need to be aware of these challenges in order to provide a safer and dignified environment for the users. Nevertheless, it is important to acknowledge that smart house systems, at some point, cannot solve all the problems that are related to ageing, disabilities and diseases. There are needs that people develop as they age and smart house technology cannot help them any more [16].

\section{Conclusion and Future Work}

In this study, activity recognition modelling (HAM) is researched. The goal is to find the normal and abnormal behaviour of the person living in a smart house. Decision trees have been used to perform activity recognition because they can predict responses to data. The output from the activity recognition task is used as an input for the modelling task.

The input data for the decision trees learning task are the rooms, duration and position. The responses are 
the activities. A total accuracy of $88.02 \%$ was achieved for activity recognition using decision trees. Thus, decision trees can be a good tool for activity recognition. However, HAM did not meet the expected results.

The possible reason for this is that decision trees do not enforce to verify every single input variable before calculating a result. Therefore, more research on how to check every variable before estimating a results needs to be studied. Alternatively, combining decision tree algorithm with another probabilistic model could be a possible solution for HAM.

\section{References}

[1] Reaz MBI. Artificial Intelligence Techniques for Advanced Smart Home Implementation. Acta Technica Corviniensis-Bulletin of Engineering. 2013;6(2):51.

[2] Vainio AM, Valtonen M, Vanhala J. Proactive fuzzy control and adaptation methods for smart homes. Intelligent Systems, IEEE. 2008;23(2):42-49.

[3] Chan M, Estève D, Escriba C, Campo E. A review of smart homes-Present state and future challenges. Computer methods and programs in biomedicine. 2008; 91(1):55-81.

[4] Alam M, Reaz M, Ali M, Samad S, Hashim F, Hamzah M. Human activity classification for smart home: A multiagent approach. In: Industrial Electronics \& Applications (ISIEA), 2010 IEEE Symposium on. IEEE. 2010; pp. 511-514.

[5] Bourobou STM, Yoo Y. User Activity Recognition in Smart Homes Using Pattern Clustering Applied to Temporal ANN Algorithm. Sensors. 2015; 15(5):11953-11971.

[6] sentralbyraa S. Key figures for the population, 2017. https://www.ssb.no/en/befolkning/nokkeltall/population. 2018. Accessed 2017-12-10.

[7] Sanchez VG, Pfeiffer CF, Skeie NO. A Review of Smart House Analysis Methods for Assisting Older People Living Alone. Journal of Sensor and Actuator Networks. 2017;6(3):11.

[8] McBurney S, Papadopoulou E, Taylor N, Williams H. Adapting pervasive environments through machine learning and dynamic personalization. In: 2008 IEEE International Symposium on Parallel and Distributed Processing with Applications. IEEE. 2008; pp. 395-402.

[9] Bao L, Intille SS. Activity recognition from user-annotated acceleration data. In: Pervasive computing, pp. 1-17. Springer. 2004;.

[10] Fan X, Huang H, Xie C, Tang Z, Zeng J. Private smart space: Cost-effective ADLs (Activities of Daily
Livings) recognition based on superset transformation. In: Ubiquitous Intelligence and Computing, 2014 IEEE 11th Intl Conf on and IEEE 11th Intl Conf on and Autonomic and Trusted Computing, and IEEE 14th Intl Conf on Scalable Computing and Communications and Its Associated Workshops (UTC-ATC-ScalCom). IEEE. 2014; pp. 757-762.

[11] Lara OD, Labrador MA. A survey on human activity recognition using wearable sensors. IEEE Communications Surveys and Tutorials. 2013; 15(3):1192-1209.

[12] Ordónez FJ, de Toledo P, Sanchis A. Activity recognition using hybrid generative/discriminative models on home environments using binary sensors. Sensors. 2013;13(5):5460-5477.

[13] Ordonez. Activities of Daily Living (ADLs) Recognition Using Binary Sensors Data Set. 2013. Accessed: 2017-05-01.

URL https :

//archive.ics.uci.edu/ml/datasets/ Activitiestof+Daily+Living+28ADLs29+ Recognition+Using+Binary+Sensors

[14] Pfeiffer CF, Sánchez VG, Skeie NO. A Discrete Event Oriented Framework for a Smart House Behavior Monitor System. In: Intelligent Environments (IE), 2016 12th International Conference on. IEEE. 2016; pp. 119-123.

[15] Chandola V, Banerjee A, Kumar V. Anomaly detection: A survey. ACM computing surveys (CSUR). 2009; 41(3):15.

[16] Sánchez VG, Taylor I, Bing-Jonsson PC. Ethics of Smart House Welfare Technology For Older Adults: A Systematic Literature Review. International Journal of Technology Assessment in Health Care. 2017;pp. 1--9. 